\title{
Within-host spatiotemporal dynamic of systemic salmonellosis: ways to track infection, reaction to vaccination and antimicrobial treatment
}

\author{
Omar Rossi $^{1 *}$, Myrto Vlazaki ${ }^{1}$, Panchali Kanvatirth ${ }^{1}$, Olivier Restif ${ }^{1}$, Pietro Mastroeni ${ }^{1}$
}

1: University of Cambridge, Department of Veterinary Medicine, Madingley Road CB3 0ES, Cambridge (UK)

*: Current affiliation: GSK Vaccines institute for Global Health, Via Fiorentina 1, 53100, Siena (Italy)

\section{Corresponding authors:}

Dr Omar Rossi, GSK Vaccines Institute for Global Health S.r.l. (GVGH), Via Fiorentina 1, 53100, Siena (Italy). e-mail: omar.x.rossi@gsk.com

Dr Pietro Mastroeni, Department of Veterinary Medicine, Madingley Road CB3 0ES, Cambridge (UK). email.: pm274@,cam.ac.uk

Keywords: Salmonella, infection, antimicrobials, vaccines.

\section{Abstract:}

During the last two decades our understanding of the complex in vivo host-pathogen interactions has increased due to technical improvements and new research tools. The rapid advancement of molecular biology, flow cytometry and microscopy techniques, combined with mathematical modelling, have empowered in-depth studies of systemic bacterial infections across scales from single molecules, to cells, to organs and systems to reach the whole organism level. By tracking subpopulations of bacteria in vivo using molecular or fluorescent tags, it has been possible to reconstruct the spread of infection within and between organs, allowing unprecedented quantification of the effects of antimicrobial treatment and vaccination. This review illustrates recent advances in the study of heterogeneous traits of the infection process and illustrate approaches to investigate the reciprocal interactions between antimicrobial treatments, bacterial growth/death as well as inter- and intra-organ spread. We also discuss how vaccines impact the in vivo behaviour of bacteria and how these findings can guide vaccine design and rational antimicrobial treatment. 


\section{Highlights:}

- Isogenic tagging, fluorescent growth reporters, single cell flow cytometry and advanced microscopy techniques have empowered in-depth studies of systemic bacterial infections from single cells to whole organism level.

- The full picture of the complex infection processes has been gained by analysis of experimental results with the help of appropriate mathematical models.

- Use of those approaches enabled to investigate the population dynamics under and upon cessation of antimicrobial treatments, and to guide vaccine treatment. 


\section{Salmonella enterica as model for the discovery of new methods and approaches to study} population dynamics during systemic infection

Despite improvements of hygienic conditions and in some cases the availability of effective antimicrobials and vaccines, bacterial diseases still pose a threat for humankind causing about six million deaths per year (MacLennan and Saul, 2014). Biological and clinical research has started to unravel the complex behaviour of bacteria during infections and their reaction to treatments, from the level of the whole animal organism down to single cells. Pathogens show higher phenotypic heterogeneity in the tissues of infected hosts compared to laboratory cultures, including bacterial growth rates, aggregation state, metabolism and stress responses (Bottery et al., 2019, Duneau et al., 2017, Gjini and Brito, 2016, Grant et al., 2008, Kolter et al., 1993, Shehata and Marr, 1971). This is, at least in part, due to the diverse microenvironments where individual bacteria reside at different times of the infection process, leading to their exposure to a variety of host cell types, immune mediators (e.g. cytokines) and cellular antimicrobial metabolites.

Systemic Salmonella enterica infections are widespread and cause diseases in humans and other animals. Typhoid fever, paratyphoid fever (Crump, 2012, Crump and Mintz, 2010) and invasive nontyphoidal salmonellosis (Crump et al., 2015, Kariuki et al., 2015)), have been the topic of extensive research, providing a paradigm for other diseases where the bacteria that have both intracellular and extracellular phases in their lifecycle. The availability of robust in vivo murine models of systemic Salmonella infection, coupled with the possibility to genetically manipulate both the pathogen and the host, allows experimentation within the complexity and biological relevance of a whole mammalian organism. The integration of several methods, described in this article, applied to this host-pathogen combination has provided an understanding of immunity, treatment, and vaccine design in humans and other animals.

\section{The interplay between bacteria and the host in vivo: population dynamics and the importance of bottlenecks}

70 Several variables determine the course and outcome of an infection process. These include, amongst others, the genetic makeup and immune status of the host, the level of virulence of the bacterium and the infecting dose. Bacteria are exposed to constrains posed by the host, and combinations of host and bacterial factors determine the progression of the disease.

Recent methods for the study of infection in vivo have enabled the mapping of spatiotemporal population dynamics of bacteria during an infection with unprecedented resolution. This has added details to our knowledge of how vaccines and antimicrobial treatments impact on the infection process often via the generation of "bottleneck events". 
Bottlenecks are constraints that may lead to reductions in the bacterial population size and/or may mechanistically or stochastically select bacterial subpopulations. Bottlenecks can be caused by anatomical barriers, immunological mechanisms, antimicrobials or vaccine-induced enhancements in host resistance. Unless they determine a marked reduction in the size of the overall viable bacterial population, bottlenecks can be difficult to detect because they can be followed by a phase of exponential expansion of surviving bacteria that are no longer inhibited by the original constraint. Surviving post-bottleneck populations may have a similar or a markedly different population structure compared to the pre-bottleneck ones, depending on the type and size of the bottleneck. Wide bottlenecks may cause only minor changes in the population size and structure of the bacteria composing the pre-bottleneck population. Tight bottlenecks can lead to situations where the prebottleneck bacterial sub-populations are differentially reduced or even lost, resulting in surviving populations that can be different from the ones present at the beginning of the infection process or in the pre-bottleneck phase/location.

In summary, canonical approaches for measuring pathogen loads in vivo (post-mortem bacterial enumeration) are not ideal to detect bottlenecks when there is no contraction of the overall bacterial population size; these unrefined approaches do not capture changes in the inherent heterogeneity of the in vivo bacterial loads within an organ or at the whole animal level. Therefore, analyses that allow tracking of individual bacterial subpopulations need to be used to gain a full picture of how the intertwined interactions between the pathogen and the host impact on the within-host evolution of the infection process.

\section{Techniques to track bacterial subpopulations and their growth status in vivo}

Capitalising on technological advances, four experimental approaches have shown great promise for studying processes of within-host population dynamics that cannot be directly observed by traditional methods: isogenic tagging, advanced microscopy, single-cell flow cytometry, and fluorescent growth reporters (Figure 1). 


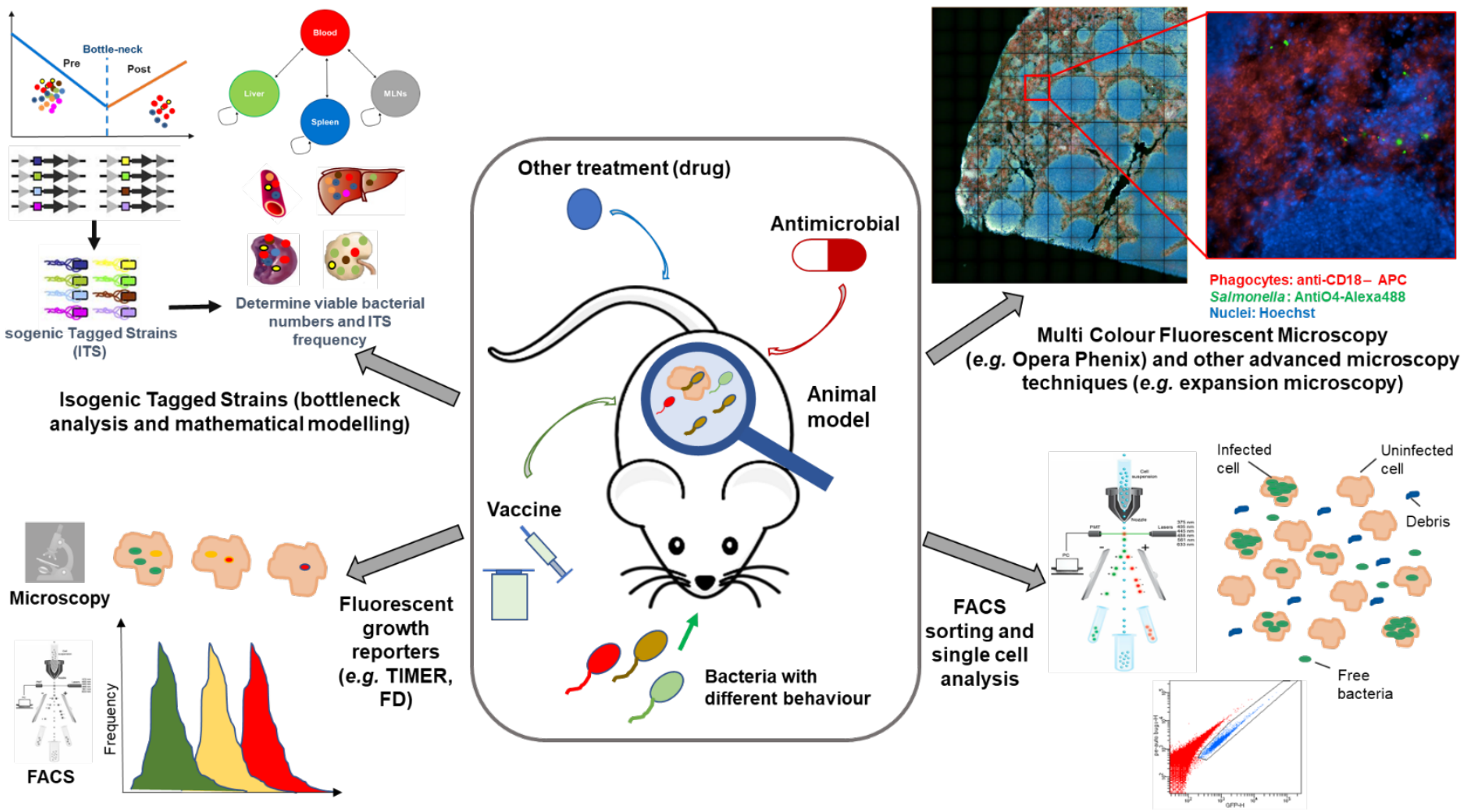

Figure 1. Techniques to track and reconstruct population dynamics at single cell level within a whole experimental animal.

3.1 Isogenic tagging - Bottlenecks in bacterial infections can be inferred by determining changes between different time-points of mixes of isogenic tagged strains (ITS) that contain inheritable distinguishable markers. These are usually specific nucleotide sequences (tags) inserted in a noncoding region of the chromosome. It is essential that the tags do not alter pathogen fitness (Grant, et al., 2008), and are uniquely detectable to allow the quantification and tracking of each tagged subpopulation in vivo. The relative frequencies of ITS within a bacterial population can be measured by quantitative PCR (Grant, et al., 2008) or sequencing of the tagged region (Rossi et al., 2017). Earlier implementations of this approach using genetic variants that could be identified by culture on selective media (with different substrates (Meynell and Stocker, 1957) or antibiotics (Moxon and Murphy, 1978)) suffered from both a lack of control of fitness differences in vivo and strict limitations in the number of strains that could be used simultaneously in an experiment. In contrast, the use of multiplex PCR or sequencing allows the monitoring of unlimited number of ITS (Abel et al., 2015, Abel et al., 2015, Schwartz et al., 2011).

Presence, absence and relative proportions of ITS in different organs, together with the absolute number of viable bacteria, can be monitored at different stages of experimental infections and treatment. This approach, combined with mathematical analyses (see below) allows to infer not just bottlenecks, but also spatiotemporal variations in the rates at which bacteria divide, die and disperse 
in multiple organs and to identify differences in behaviour of the bacterial population at different stages of the infection and/or treatment process. For example, increases in heterogeneity within a particular anatomical site are considered as an indication of amplification and/or preferential selection of a restricted number of bacterial subpopulations; reductions in heterogeneity between two different anatomical sites can reveal the transfer of bacteria between the two sites. Bacterial death rates can be estimated from the disappearance coupled with absolute load decrease of ITS through time, and growth by the appearance and relative increase of different ITS through time (Grant, et al., 2008). Similarly, the loss of some of the ITS in a defined population would indicate that a certain proportion of bacteria have died and/or have been replaced by replication of some other subpopulation (Figure 1, top left panel).

3.2 Advanced microscopy techniques - Multicolour fluorescence microscopy (MCFM) and confocal microscopy have been used to gather information on the location of the bacteria in the tissues, to identify and characterise the sites and the cells where Salmonella is found and to determine the number of bacteria within each infected cell (Grant et al., 2012). This enables to determine the intraversus extra-cellular location of the bacteria, their association with inflammatory multicellular pathological lesions (Sheppard et al., 2003), as well as the location and cell surface markers of the infected cells in tissue samples. This is achieved by staining with fluorescently marked antibodies specific for host cell markers and/or bacteria (Sheppard, et al., 2003), and/or using bacteria engineered to express fluorochromes (e.g. GFP) (Rollenhagen et al., 2004). However, in some phases of infection or treatment (e.g. after prolonged antimicrobial treatment) low (e.g. less than 1000 bacteria per organ) bacterial numbers in the organs would require observation of impractically large volumes of tissue to visualize sparse bacterial populations (Rossi, et al., 2017). Nowadays, more advanced technologies are available for detecting low bacterial numbers in tissues. For example, the Opera Phenix HighContent Screening fluorescence/confocal system with the Harmony high-content analysis software performs fully automated scans of large numbers of tissue sections and multicolour image acquisition, with the option of intelligent image analysis and higher magnification/resolution re-scan (Fraietta and Gasparri, 2016).

To overcome the need for culling of experimental animals at each time point before sampling, luminescent imaging can be also used to directly detect emitted photons from lux-expressing Salmonella in live anesthetized mice and therefore follow the course of infection in various organs. However, bioluminescence approaches lack sensitivity and resolution, with a minimum of hundreds of bacteria clustered together being required to obtain detectable signals in vitro (without the added 
loss of signals due to skin and tissue), thus limiting the possibility of quantifying the dynamics of small subpopulations of bacteria in vivo (Avci et al., 2018).

Expansion microscopy is another powerful technique to detect bacteria in small organs (e.g. lymph nodes) or in specific phases in which their overall number is low. This technique relies on the expansion of small structures using an isotropic polymer system (and clarification of the organs if necessary to reduce autofluorescence), still maintaining the same nanoscale detail of the organ. The expanded structure can be then visualized at the same level of detail with a lower magnification. In other words, expansion microscopy enables high-resolution imaging on a conventional light microscope (Lim et al., 2019). Another interesting and novel technique is represented by superresolution stochastic optical reconstruction microscopy (STORM)-correlative light electron microscopy (CLEM); this allows bacteria to be imaged within host cells with a resolution of $20 \mathrm{~nm}$ (van Elsland et al., 2018).

In parallel to advancements in the resolution of microscopy techniques, algorithms are continuously developed (e.g. Harmony software of Opera Phoenix) to map the spatial location of sparse bacterial populations and to initiate high-resolution imaging protocols via confocal, two-photon and structured-illumination microscopy in situ, thus automatizing the microscopy systems (Figure 1, top right panel).

3.3. Single cell flow cytometry - Global genomic, proteomic and transcriptional profiling can be nowadays performed at the single-cell level (Mosser and Edwards, 2008, Saliba et al., 2016, Westermann et al., 2012) after FACS-sorting the cells of interest. This enables to study the heterogeneity of individual microbe-host cell interactions during infection. For example, combining FACS-sorting of infected cells with single-cell RNA sequencing has revealed that macrophages infected by non-growing bacteria display a pro-inflammatory M1 state, whereas macrophages containing fast-growing bacteria were predominantly in an anti-inflammatory M2 state (Saliba, et al., 2016). This is an important finding that reveals how heterogeneity in pathogen behaviour is intertwined and possibly determines heterogeneity in the phenotype of the infected host cell. Single cell flow cytometry enables to compare the level and type of specific markers in different organs, at different stages of treatment or infection, or to compare the phenotype of infected and noninfected cells. Furthermore, simultaneous determination of the transcriptomic profile (dual RNA-seq) of both bacteria and the host (Stapels et al., 2018, Westermann et al., 2016) can provide information about the interface between bacteria and host (Oshota et al., 2017). The main drawbacks of these techniques are the cost, the amount of data generated, and sometimes the lack of specific behaviour in the context of the whole organ/organism (Figure 1, bottom right panel). 
3.4 Fluorescent growth reporters - Single-cell fluorescent growth reporters (Helaine et al., 2010) have been successfully used to measure bacterial division rates in vivo, and reveal the presence of distinct Salmonella subsets with divergent division rates in different tissues. Here we outline two methods that differ in the information they provide: i) Fluorescence Dilution (FD) analysis tracks the number of generations since the start of infection; ii) the TIMER reporter reveals the recent rate of division at the time of harvesting.

FD analysis (Helaine et al., 2014, Helaine and Holden, 2013, Helaine, et al., 2010) is used to detect bacteria that have not divided or that have undergone a limited number of divisions from the beginning of the infection to the time of observation. This can be done exploiting fluorescent proteins that are produced only in the presence of inducers (e.g. arabinose or IPTG); when the bacteria replicate in the absence of the inducer (e.g. arabinose), a rapid decrease in signal ("dilution") of the preformed pool of fluorescent protein is observed. Therefore, the measurement (e.g. by FACS) of reduction of fluorescence intensity over time is a proxy of the number of replications undergone by the bacterium. Hence, bacteria with low and high fluorescence respectively represent cells that have undergone multiple or no/few rounds of cell division (Helaine, et al., 2010).

TIMER (DSredS197T) protein spontaneously changes fluorescence colour (e.g. emission spectra) from green to orange over time. Although the complex atomic and molecular underlying processes are not fully understood, variations in colour of fluorescence can be accurately calibrated to measure the current division rate of bacteria at the time of observation (Claudi et al., 2014). In cells that have recently undergone cell division, the fast maturing green TIMER molecules are more abundant compared to slowly maturing orange TIMER molecules, resulting in a dominant green fluorescence (Claudi, et al., 2014). In contrast, in non-proliferating cells, the slowly maturing orange TIMER molecules accumulate, yielding a characteristic green/orange fluorescence.

A major strength of both FD and TIMER markers is that they can be used at the single-cell level to reveal phenotypic heterogeneity, either by microscopy or by flow cytometry (FACS). FACS also allows to sort single host cells based on the growth reporter or other markers, and their transcriptional profile can then be determined by scRNA-seq (Saliba, et al., 2016). Both FD and TIMER have been used in vivo to study the growth history of Salmonella during antimicrobial treatment. This has revealed that non-replicating Salmonella survive exposure to antibiotics better than fast-dividing bacteria. However, non-replicating bacteria are normally a minority of the overall population in the organ and therefore the most abundant population that remains in the tissues after treatment consists of partially antibiotic-tolerant, moderately-growing bacteria (Claudi, et al., 2014). Additional applications for FD or TIMER are their combination with microscopy (Claudi, et al., 2014), also envisaging potential combinations with partially automated microscopy (like the Opera Phenix) to 
determine and visualize the distribution of bacteria with different division history in the organs (Figure 1, bottom left panel).

One of the main limitations of FD is the number of generations that can be followed over time (6 to 10 generations), as the signal decrease rapidly with a small number of bacterial generations; the latter is efficiently overcome with TIMER approaches that rely on expression under promoters highly active in vivo (Rollenhagen, et al., 2004). However, a different limitation of TIMER is the fact that maturation kinetics of the fluorescent reporters depend on oxygen partial pressure (Claudi, et al., 2014), potentially limiting the use of the technique to study bacterial dynamics in homogeneously oxygenated tissues such as the spleen.

\section{The importance of mathematical modelling}

One attraction of the experimental methods outlined above is that they can produce simple semiquantitative patterns that can be intuitively interpreted (Crimmins and Isberg, 2012). For example, a loss of ITS diversity is the signature of a bottleneck; using FD, a subset of bacteria with constant fluorescence intensity represents persister cells. Essentially, ITS are reporters of bacterial mortality and migration, whereas FD and TIMER are markers of bacterial replication. During the course of infection however, all three processes occur simultaneously and vary in different ways within and between organs and in response to treatments. Hence simple data analysis is not sufficient to disentangle these processes: mathematical models are necessary to obtain the full picture (Vlazaki et al., 2019). Here we outline the use of models for ITS studies, but it is worth noting that similar methods can be applied to FD experimental data.

Mathematical models simulate the growth, death and within-host migration of bacteria, generating synthetic data that can be directly compared to experimental datasets. Statistical tools can then be used to "fit" the model to data, which means estimating the values of unknown parameters (division rates, death rates, migration rates) that minimise the difference between the synthetic data and a given experimental dataset. Technically, this can be done using maximum likelihood (Coward et al., 2014, Kaiser et al., 2013), Bayesian inference (Dybowski et al., 2015) or minimisation of Kullback-Leibler divergence (Price et al., 2017).

Importantly, those models do not simulate immune dynamics or antibiotic pharmacokinetics, as those variables are rarely measured in experimental infections. Instead, the models allow to estimate the impact of immunity or drugs on the division and survival rates of bacteria, by comparing the values between experimental groups or between time points. For example, it was shown that a combination of bacterial death and rapid replication leads to the colonisation of the liver and spleen by independent bacterial subpopulations in the systemic phase of Salmonella infections (Grant et al., 2008), while the 
gut-lymphoid tissue interface was found to act as a major bottleneck in the enteric phase due to the low chance of bacteria migrating from the gut to the caecal lymph node (Kaiser et al., 2013). Individual components of the host immune system have also been identified as key players in the early dynamics of infection through comparison of the killing rates between immunocompetent mice and gene-targeted mutant mice lacking specific immunological pathways (Kaiser et al. 2013; Grant et al., 2008; Coward et al., 2014).

Beyond exploratory research to characterise previously unchartered territories, other studies have used mathematical modelling integrated with experimental data to compare the dynamics between differentially treated groups. For instance, a comparison between the effects of different vaccine formulations on the within-host dynamics of $S$. enterica has been enabled through mathematical inference and quantification of the underlying dynamics (Coward et al., 2014), while comparing the dynamics of in vivo adapted versus culture-harvested bacteria revealed that the former grow faster as a result of lower death rates compared to culture-harvested bacteria (Dybowski et al., 2015). These models are stochastic, representing whole numbers of bacteria undergoing bottlenecks that can randomly wipe out some ITS and allow other to grow back, mimicking the random variations observed across replicates in the experiments (Grant, et al., 2008). In addition, different sources of measurement noise and error (e.g. due to finite sample sizes or cut-off points for quantitative PCR) can be included in the model, ensuring that parameter estimates are produced with reliable confidence intervals (Coward, et al., 2014, Price, et al., 2017). Unfortunately, the in vivo studies published so far have only used mathematical models to address the very early dynamics of infection. This pattern is not due to the lack of interest for the subsequent stages of the infection; it is the byproduct of computational limitations inherent in the likelihood inferential framework when applied to complex multiparametric systems evolving over longer time periods. These technical obstacles can be overcome with the development of computationally efficient tools like the moments-based, divergence minimisation approach (Price et al., 2017).

Finally, mathematical models could be helpful not only to analyse the data generated experimentally, but also to optimise the design of future experiments that can be carried out in a more rational and cost-efficient way, or to validate the mathematical model itself. For example, based on model predictions, unlikely scenarios can be discarded a priori and research can delve on plausible hypotheses; numbers of experimental animals, experimental interventions and measured variables can be decided in line with post-experimental data analysis. Full implementation of proper mathematical models ultimately should reduce animal experimentation and allow in silico determination of treatments' output. 
The techniques and approaches described above are tools to study in vivo infections. Below we will delve on some areas of infection biology that have benefitted from the multidisciplinary integration of these techniques and approaches. We will briefly illustrate some representative examples of findings that would have been otherwise impossible to achieve with compartmentalized research.

\subsection{Understanding spread and distribution of the infection.}

Salmonella causing invasive diseases (like invasive non-typhoidal salmonellosis or typhoid fever) invade the gut and reach the bloodstream, with a proportion of the bacteria passing through the mesenteric lymph nodes (MLNs) (Carter and Collins, 1974). Lymph nodes are central in the process, with speed of onset of infection in various organs being different (Awofisayo-Okuyelu et al., 2019). Experimental infections of mice with a discrete number of ITS have revealed that the population structure present in the lymphatic compartment throughout the infection is markedly different from the one present in other systemic organs (e.g. spleen and liver have common population structure); thus MLNs are a separate compartment within the complexity of a multiorgan mammalian host (Rossi, et al., 2017). In blood, bacteria can be found either extracellularly or associated with leukocytes (Vazquez-Torres et al., 1999). From blood, bacteria reach the liver, spleen and bone marrow where they infect resident phagocytes (principally red pulp and marginal zone macrophages in the spleen and Kupffer cells in the liver (Richter-Dahlfors et al., 1997, Salcedo et al., 2001)). Confocal microscopy reveals that the infection begins at an intracellular density of one bacterium per host cell; then inflammatory mononuclear cells are recruited to the sites of infection with the formation of multicellular pathological lesions that trap the bacteria within discrete foci, surrounded by normal tissue (Richter-Dahlfors et al., 1997). This process is mediated by cytokines and adhesion molecules, with TNF- $\alpha$ being crucial, as its absence results in the lack of formation or regression of multicellular pathological lesion and causes recrudescence of the infection (Clare et al., 2003, Everest et al., 1998, Mastroeni et al., 1991, Mastroeni et al., 1995, Mastroeni et al., 1992, Mastroeni et al., 1993, Rossi et al., 2017, Withanage et al., 2005). Cytokine networks are also essential for the activation of the recruited cells and for the expression of their antimicrobial activity (Mastroeni et al., 1999, Mastroeni et al., 1996, Mastroeni et al., 1998, Muotiala and Makela, 1990). Within multicellular pathological lesions reactive oxygen and nitrogen species (ROS and RNS) restrict intracellular growth (Grant, et al., 2008, Mastroeni et al., 2000, Vazquez-Torres et al., 2000, Vazquez-Torres et al., 2000). The kinetics of intracellular growth is dependent upon the balance between host-resistance function/genetics and the virulence of the bacterial strain (Mastroeni and Grant, 2013). 
One of the key features of the spread and distribution of Salmonella in tissues, revealed by fluorescence microscopy approaches, is represented by low intracellular numbers of bacteria per infected cells regardless of the net growth rate of the bacteria. Growth of the bacteria in the tissues is paralleled by an increase in the number of infected cells and multicellular pathological lesions and not by a substantial increase in the intracellular number of bacteria within each infected host cells. The intracellular densities of Salmonella in the organs follow a Poisson distribution skewed towards low numbers underpinned by bacterial escape from infected phagocytes and dissemination to 335 uninfected cells to form new infection foci (Sheppard, et al., 2003). Reinfection events occur at a very low rate $(<1 \%)$ (Gog et al., 2012) and new foci of infection are largely clonal (Sheppard, et al., 2003). Bacteria with disabling mutations in genes of the Salmonella pathogenicity island-2 (SPI-2) encoded Type III secretion system are impaired in tissue spread and accumulate intracellularly within few cells (Grant, et al., 2012).

340 During the extracellular phase Salmonella become vulnerable to antibodies and complement (Rossi et al., 2019) that opsonise the bacteria and target them to receptors on the surface of phagocytes increasing the ROS-dependent antimicrobial functions of these cells (Liang-Takasaki et al., 1983, Mastroeni and Grant, 2013).

Performing infections of the mouse with low numbers of ITS, it has been possible to outline the whole-body dynamics in the early stages of systemic disease. In the initial phase of infection (first 6 hours after inoculation), concomitant death and rapid bacterial replication leads to the establishment of independent bacterial subpopulations in different organs that spread only locally. The subpopulation structure (presence and relative proportion of individual ITS in each organ) remains different in different organs up to 24 hours post infection (Grant, et al., 2008). This is an intriguing 350 finding; in fact, early in infection the bacteria spread from cell to cell at distant sites in each organ, but their spread appears to be confined to the boundaries of a particular organ or tissue. With the progression of the infection, decreased microbial mortality leads to an exponential increase in the number of bacteria within the spleen and liver, that is paralleled by the spread of the microorganisms throughout the body via blood and establishment of new infection foci, with the mixing of bacteria between organs via bacteraemia and further stochastic selection (Grant, et al., 2008).

The combination of ITS and gene targeted immune-deficient mice has added an additional layer of resolution to the study of the interactions between bacteria and the host. This approach has allowed to interrogate the precise function of individual elements of the immune response on the growth, death and spread of the infection. For example, the use of ITS in mice lacking the gp19 subunit of the phagocyte NADPH oxidase conclusively established the bactericidal activity of reactive oxygen metabolites on intracellular Salmonella in vivo (Grant, et al., 2008). With the use of FD, replication 
has been directly quantified, and the role of SPI-2 T3SS has been shown mainly in fostering bacterial replication, but not in avoidance of killing by macrophages. FD was also used to understand that nonreplicating bacterial cells are generated immediately after uptake by macrophages; in fact the vacuolar acidification and nutritional deprivation within SCV induce gene expression in the bacteria with activation of several toxin-antitoxin modules, the creation of phenotypic heterogeneity and formation of persistor cells acting as reservoir for infections able to resume their division when environmental conditions becomes more favourable (Helaine, et al., 2014, Helaine, et al., 2010). In conclusion, combining multidisciplinary approaches can reveal the true dynamics of the infection process. These approaches include acquisition of distributional data obtained from the direct observation of bacterial populations in tissues by microscopy, the use of isogenic tagged strains, mathematical modelling, fluorescent reporters of bacterial division history, immunological single cell techniques such as flow cytometry, and transcriptomic profiling of tissues and single cells.

\subsection{Population dynamics and antimicrobial treatment}

Currently, little is known about how antibiotics act in vivo at the level of individual bacteria and individual organs/host cells. This is a neglected area of in vivo infection biology that will require interdisciplinary and comprehensive efforts and dedicated funding.

Intense research efforts have focussed on the prevalence and spread of antimicrobial resistance (AMR) genes/phenotypes in microbial species. However, difficulties in treating bacterial infections cannot always be ascribed to pathogens carrying antimicrobial resistance genes. Treatment of an infection with antibiotics does not always result in the rapid and complete elimination of the pathogen from the tissues even when the bacteria are highly susceptible to the drug in vitro.

When Salmonella systemic infections are treated with antimicrobials such as beta-lactams or fluoroquinolones a bi-phasic reduction in bacterial numbers is observed with an initial rapid decrease in the bacteria load followed by a phase of slower reduction in bacterial numbers (Claudi, et al., 2014, Kaiser, et al., 2013, Rossi, et al., 2017). This is often followed by a third phase where the number of culturable bacteria remains constant in the tissues despite the prolongation of the treatment and the fact that these persisting bacteria retain susceptibility to the drug. In fact, when bacteria are isolated and cultured from animal tissues during and/or after antibiotic treatment, they do not show a decrease in antibiotic sensitivity in vitro (as measured by the MIC [Minimum Inhibitory Concentration]). The persistence of antimicrobial-sensitive bacteria during and after treatment is a problem of great medical importance as it can lead to chronic infections, emergence of antimicrobial resistant populations, prolonged transmission, within-host bacterial evolution and dangerous relapses especially in immune-deficient individuals (Crump et al., 2004, Gordon, 2011, Klemm et al., 2016, 
Okoro et al., 2012). Notably, persistence of non-AMR resistant bacterial population occurs even in the presence of an intact immune system (Casadevall and Pirofski, 2000, Gordon et al., 2010, Kaiser, et al., 2013, Klemm et al., 2016, Mc, 1958, Okoro, et al., 2012). Currently, the efficacy of a particular treatment is still difficult predict a priori and there is no guarantee that an individual is going to clear the infection after a course of antimicrobials.

The reasons for discrepancies between the efficacy of some antibiotics in vitro and poor outcome of treatment in vivo are difficult to explain using traditional pharmacokinetic and pharmacodynamics parameters. Methods that capture the replicative status and location of different bacterial subpopulations within organs and tissues as well as the immunological status of the host allow consideration of key variables within the links between treatment and outcome of an infection (Grant et al., 2009, Grant, et al., 2008, Sheppard, et al., 2003).

\subsection{Replicative status and heterogeneity}

Subpopulations of bacteria have extensive phenotypic variation in in vivo fission rates, ranging from fast division to a non-replicating intracellular status within phagocytes. Therefore, studying the correlations between growth rates and antibiotic treatment is important to understand the efficacy of antimicrobial treatments as well as why and where bacteria may persist in the tissues. As briefly mentioned above, studies in mice revealed that slowly-replicating bacterial populations are less susceptible to antimicrobials; these non-replicating bacteria can be completely refractory to treatment but can resume growth in the absence of antimicrobial exposure (Claudi, et al., 2014, Gordon, et al., 2010, Helaine, et al., 2014, Helaine and Holden, 2013, Helaine, et al., 2010, Klemm, et al., 2016, Maskell and Hormaeche, 1985, Maskell and Hormaeche, 1986, Okoro, et al., 2012). Slow-growing bacterial subpopulations can have a selective advantage under antimicrobial pressure (Claudi, et al., 2014, Helaine, et al., 2014) and prolonged treatment with antimicrobials can kill up to $99 \%$ of bacteria from the tissues leaving a residual microbial load that is able to cause relapse (Bonina et al., 1990, Maskell and Hormaeche, 1985).

Using integrated techniques such as use of fluorescent bacterial strains, microscopy and a mathematical analysis, it has been observed that during systemic infections bacterial populations undergo a phenotypic switch to enter into a slow-growing or growth-arrested phase which is phenotypically tolerant to antimicrobial action (Balaban et al., 2004, Kussell and Leibler, 2005). This phenotypic divergence has been observed by using fluorescent intracellular growth reporters that are diluted in the bacterial cytoplasm at every division (Fisher et al., 2017); almost all bacteria extracted form MLNs after multiple days of treatment retained very high fluorescence levels (e.g. similar to the fluorescence level detected at the beginning of the experiment), indicating that the subpopulations of 
430 Salmonella capable of surviving treatment in mice undergo none or very few cell divisions (Helaine, et al., 2014). A similar behaviour was confirmed in spleen with fast-growing Salmonella subpopulations being extensively killed and slow growing bacteria surviving the treatment (Claudi, et al., 2014).

Toxin-antitoxin modules, stress responses, host cell microenvironment, and nutrient deprivation, are mechanisms involved in the formation of persisters (Claudi, et al., 2014, Helaine, et al., 2014). Intriguingly, persisting bacteria that resist to antibiotics still retain the ability to transcribe, translate and translocate SPI-2 virulence effectors in a non-growing state, when internalized in macrophages; this enables the reprogramming of host cells from a M1-like pro-inflammatory bactericidal state to a M2-like anti-inflammatory permissive state, a critical step for persisters to survive long term in macrophages (Pham et al., 2020). It has also been shown using a combination of flow cytometry, fluorescence microscopy and RNA seq that non-growing persisters use the effector SteE from the SPI-2 T3SS to direct macrophages to the M2 state to allow them to persist and remain tolerant to antimicrobials(Stapels, et al., 2018).

The dynamics of bacterial persistence are different in different organs and tissues. Salmonella can be detected in spleens, livers and MLNs organs of mice for more than 10 days post-infection and antimicrobial treatment. MLNs are a peculiar niche for persistence (Griffin et al., 2011, Kaiser et al., 2014, Rossi, et al., 2017). In fact, a reduction in viable bacterial numbers is not obvious in MLN as a consequence antimicrobial treatment. The overall bacterial load in these tissues is not affected by antimicrobial treatment (Claudi, et al., 2014, Rossi, et al., 2017). Other studies also indicate that 450 bacteria in the MLNs are lest affected by antibiotic treatment compared to those residing in other tissues. In fact, bacteria become largely undetectable within a few days of exposure to antimicrobials by direct in vivo imaging of Salmonella lux positive, and MLNs remain the final organ to display a detectable signal of infection (Griffin, et al., 2011). Consistently, following antimicrobial cessation, the earliest detection of bacteria also occurred in MLNs (hence a site of rapid relapse), with bacterial growth eventually progressing to include multiple systemic tissues, most notably the spleen and liver. Cessation of treatment results either in the relapse of the infection or in prolonged persistence of the bacteria in defined niches, depending on host genetics and/or the virulence of the bacterial strain. In situations where genetically susceptible experimental animals are infected with virulent bacteria the infection relapses promptly if treatment is withdrawn and the rate of net bacterial growth in the posttreatment phase is very similar to that of the pre-treatment phase. In this experimental setup, relapse can be avoided only if treatment is prolonged, presumably until the onset of acquired immunity in the host (Maskell and Hormaeche, 1985, Maskell and Hormaeche, 1986, Rossi, et al., 2017). Postantibiotic persistence and relapses of Salmonella infections have also been described in humans, 
especially in patients with immune-deficiencies (Gordon, et al., 2010, Klemm, et al., 2016, Okoro, et al., 2012).

The use of ITS in experimental systemic infections (Kaiser, et al., 2013, Rossi, et al., 2017) has allowed to study the subpopulation dynamics of a Salmonella infection during the treatment and relapse phase. It has become clear that the population heterogeneity of the bacteria load is not reduced during treatment indicating that the antibiotic does not select for one of few clones within the bacterial load. Furthermore, no preferential amplification of any of the ITS can be detected during the relapse phase, further indication that relapse does not originate from a restricted number of subpopulations that has been preferentially selected by the antibiotic treatment. Chronic and relapsing infections also arose evenly from the persistence of multiple subpopulations of Salmonella. (Rossi, et al., 2017).

\subsection{Location and the immune system}

Host immunity can potentially exert opposing effects on the efficacy of antibiotic treatment. On one side it co-operates with antimicrobials by eliminating bacteria from the tissues and preventing the relapse of the infection. On the other, hand immunity can limit the efficacy of antimicrobials by creating environments hostile to their penetration and efficacy (e.g. granulomas). Furthermore, phagocyte activation can determine a reduction in bacterial division rates and can activate MDR pumps, thus reducing susceptibility to many antimicrobials (references).

Granulomas can represent sites where antibiotics penetrate poorly and could therefore enable bacterial persistence during the course of treatment, with the use of microscopy and growth curves it has also been seen that cytokines such as $\mathrm{TNF} \alpha$ are required for the formation of granulomas in many bacterial infections (Mastroeni, et al., 1995). Salmonella Typhimurum infected granulomas predominantly consist of macrophages, with a mixture of both M1 and M2 stages depicting both the protective nature of a granuloma to the host while potentially being permissive to persistence of the bacteria. (Pham, et al., 2020).

Treating mice with anti-TNF $\alpha$ antibodies inhibits or reverses the formation of granulomas, and therefore could potentially enhance the efficacy of treatment against bacteria that localise within these pathological tissue lesions (Mastroeni, et al., 1995). However, as explained below, experimental evidence suggests that this may not be the case. In fact, anti-TNF $\alpha$ treatments in systemic murine Salmonella infection neither preclude nor enhance the efficacy of antimicrobial treatment, but dangerously leave the host prone to more severe relapses of the infection due to immunosuppression.

495 Combinations of anti-cytokine compounds and antimicrobial molecules may therefore not be a useful approach to treat persistent infections with intracellular bacteria like Salmonella (Rossi, et al., 2017). 
To fully understand post-antibiotic persistence in vivo it is important to indentify the host cells associated with the microorganisms during and after antimicrobial treatment. Using GFP-expressing bacteria and flow cytometry recent studies have determined the main types of splenocytes where Salmonella in found in mice during and after ciprofloxacin treatment. Dendritic cells, macrophages and neutrophils were found to be the main cell populations associated with bacteria that persist after ciprofloxacin treatment. These data extend previous studies where neutrophils had been reported as a splenic "safe" site where Salmonella persists after treatment with gentamicin (antimicrobial acting mainly on extracellular bacteria) and where dendritic cells had been reported as a site of persistence in the MLNs of mice in an enteritis model (Kaiser, et al., 2013, Rossi, et al., 2017).

Upon cessation of antibiotic treatment, the expected relapse of the infection leads to the major cell types associated with Salmonella differing from the pre-treatment phase. In fact, upon re-growth in vivo, the bacteria did not re-populate spleen cells in the same proportions as in the pre-antibiotic phase (Kanvatirth et al., 2020). These data show that the cellular dynamics of the infection in the preantibiotic phase are different from the relapse phase.

The role of host cell populations in modulation the efficacy of antibiotics can be studied by depleting individual host cell types during treatment of an infection. In murine Salmonella infections, host cell depletion experiments revealed a dichotomous role of immune cell populations in the clearance or persistence of Salmonella during antimicrobial treatment (Kanvatirth, et al., 2020). In vivo depletion of PMNs resulted in higher bacterial numbers in the spleen at the end of treatment, consistent with the fact that PMNs are one of the cells principally involved in innate immunity to Salmonella and are efficient producers of ROS. While, depletion of macrophages and dendritic cells had a beneficial effect on the antimicrobial treatment, resulting in lower bacterial numbers in the spleen. Therefore, cell populations that are crucial to the control of the infection in the phase of innate immunity and that are involved in antigen presentation and generating an adaptive response are also responsible for carriage of the residual bacterial population after ciprofloxacin treatment. There is potential that by immune-modulatory treatments (Kanvatirth, et al., 2020, Kaufmann et al., 2017) and exploring unexploited enzymes as targets for antimicrobials (Becker et al., 2006) could improve the treatment of a systemic Salmonella infection.

\subsection{Population dynamics after vaccination}

The use of appropriate methods to understand the pathogenesis and in vivo infection dynamics is crucially important in the rational design of vaccination strategies and in the understanding of their potential impact on the infection process. 
530 The dispersive nature of infection caused by Salmonella has profound implications for understanding the immunological requirements of vaccine-mediated protective immunity. During the intracellular phase of growth/persistence it is plausible to postulate that immunity produced through vaccination needs to potentiate the antimicrobial action of phagocytes by Th1 type T-cell immunity. In contrast, during the extracellular phase, bacteria are vulnerable to antibody-mediated complement killing (serum bactericidal activity) (Micoli et al., 2018) and cell-mediated killing (opsonophagocytosis) (Ramachandran et al., 2016). The relative importance of these two antibody-mediated killing mechanisms in vivo varies, and it is likely to be dependent on the bacterial strain and the anatomical compartment where the bacteria are located, as well as on the relative abundance and qualitative traits (e.g. isotypes, affinity, FcR usage and complement activation) of antibodies (Rossi, et al., 2019). (Goh et al., 2011, Goh et al., 2016, Gondwe et al., 2010, MacLennan et al., 2008).

The combination of in vivo measurements in overall bacterial numbers combined with tracking of ITS and mathematical modelling has allowed an insight into how different classes of vaccines and branches of the immune response protect against secondary Salmonella enterica infections in mice (Coward, et al., 2014). These studies revealed that live attenuated Salmonella vaccines significantly reduce bacteraemia during a secondary challenge and can contain the growth and spread of the bacteria in systemic organs. Live vaccine immunisation enhances both the bacterial killing in the very early stages of the infection and the bacteriostatic control over the first day post-challenge. In contrast non-living vaccines, while able to enhance initial blood clearance and killing of virulent bacteria, do not alter the subsequent bacterial growth rate in the systemic organs, do not prevent the resurgence of bacteraemia, and fail to control the spread of the bacteria in the body (Coward, et al., 2014).

The experimental evidence that during a systemic secondary infection antibodies alone, without $\mathrm{T}$ cellular immunity, cannot control bacterial growth, spread and bacteraemia, provides an explanation for the high incidence and recurrence of systemic salmonellosis in individuals where co-morbidities such as malaria and HIV impair cell-mediated immunity (T-cells and phagocytes) (Mastroeni and Rossi, 2016).

Vaccination can also affect the occurrence of persisters in vivo. Vaccination of mice with killed $S$. Typhimurium results in a reduction in bacterial persisters of about 500-fold as compared to naïve mice upon secondary infection and ciprofloxacin/ampicillin treatment. Furthermore, vaccination prevents the transfer of transconjugants, hence suggesting a central role that vaccines may have not only to protect against infections, but also to prevent the spread of anti microbial resistance (AMR) (Bakkeren et al., 2019).

\section{Conclusion and perspectives}


Understanding the in vivo dynamics of bacterial persistence at the single cell level during antimicrobial treatment and the niches where persistent bacteria reside after therapy is of paramount importance for developing targeted medical and veterinary treatments complementary or alternative to classical antimicrobial treatment based on manipulation of the host (Czaplewski et al., 2016, Hancock et al., 2012). This is crucial considering the slow progress in current pipelines for discovery of new antimicrobial molecules (Lewis, 2013). These will include designing host-cell specific immunological approaches, as shown by the different effects of cell-depletions in vivo, or in the future delivering drugs "in situ" at the infection foci to the right cells where persistent bacteria are found. This will maximise the therapeutic potential of currently available antimicrobials and will inform about the requirement for new drugs and new targeted delivery systems (Kaufmann, et al., 2017, Lewis, 2013). Similarly, an in-depth understanding of the mechanism of action of vaccines and antibodies induced on the bacterial subpopulations will allow the maximisation and the design of vaccines that would target specific cell types, with the right antigen and the right adjuvant for each specific site.

All the above can truly be performed only by maximising and integrating all the information that is available with sophisticated techniques described in this review both at the single-cell and whole organism level.

\section{Ethics}

All animal studies were ethically reviewed and carried out in accordance with European Directive 2010/63/EEC and the GSK policy on the Care, Welfare and Treatment of Animals.

\section{Authors contribution}

All authors contributed on writing/original draft preparation, review and editing. All authors have read and agreed to the published version of the manuscript.

\section{Sponsorship and funding}

University of Cambridge (UK).

\section{Conflict of interest}

ORo is currently an employee of the GSK Vaccines Institute for Global Health (GVGH), part of the GSK group of companies. This does not alter the authors' adherence to all Journal policies on data and material sharing. 


\section{References}

Abel, S., Abel zur Wiesch, P., Chang, H.H., Davis, B.M., Lipsitch, M., Waldor, M.K., 2015. Sequence tag-based analysis of microbial population dynamics. Nat Methods. 12, 223-226, $223 \mathrm{p}$ following 226.

Abel, S., Abel zur Wiesch, P., Davis, B.M., Waldor, M.K., 2015. Analysis of Bottlenecks in Experimental Models of Infection. PLoS Pathog. 11, e1004823.

Avci, P., Karimi, M., Sadasivam, M., Antunes-Melo, W.C., Carrasco, E., Hamblin, M.R., 2018. Invivo monitoring of infectious diseases in living animals using bioluminescence imaging. Virulence. 9, 28-63.

Awofisayo-Okuyelu, A., Pratt, A., McCarthy, N., Hall, I., 2019. Within-host mathematical modelling of the incubation period of Salmonella Typhi. R Soc Open Sci. 6, 182143.

Bakkeren, E., Huisman, J.S., Fattinger, S.A., Hausmann, A., Furter, M., Egli, A., Slack, E., Sellin, M.E., Bonhoeffer, S., Regoes, R.R., Diard, M., Hardt, W.D., 2019. Salmonella persisters promote the spread of antibiotic resistance plasmids in the gut. Nature. 573, 276-280.

Balaban, N.Q., Merrin, J., Chait, R., Kowalik, L., Leibler, S., 2004. Bacterial persistence as a phenotypic switch. Science. 305, 1622-1625.

Becker, D., Selbach, M., Rollenhagen, C., Ballmaier, M., Meyer, T.F., Mann, M., Bumann, D., 2006.

Robust Salmonella metabolism limits possibilities for new antimicrobials. Nature. 440, 303-307.

Bonina, L., Carbone, M., Matera, G., Teti, G., Joysey, H.S., Hormaeche, C.E., Mastroeni, P., 1990.

Beta-lactam antibiotics (aztreonam, ampicillin, cefazolin and ceftazidime) in the control and eradication of Salmonella Typhimurium in naturally resistant and susceptible mice. The Journal of antimicrobial chemotherapy. 25, 813-823.

620 Bottery, M.J., Passaris, I., Dytham, C., Wood, A.J., van der Woude, M.W., 2019. Spatial Organization of Expanding Bacterial Colonies Is Affected by Contact-Dependent Growth Inhibition. Curr Biol. 29, 3622-3634 e3625.

Carter, P.B., Collins, F.M., 1974. The route of enteric infection in normal mice. J Exp Med. 139, 1189-1203.

625 Casadevall, A., Pirofski, L.A., 2000. Host-pathogen interactions: basic concepts of microbial commensalism, colonization, infection, and disease. Infection and immunity. 68, 6511-6518.

Clare, S., Goldin, R., Hale, C., Aspinall, R., Simmons, C., Mastroeni, P., Dougan, G., 2003. Intracellular adhesion molecule 1 plays a key role in acquired immunity to salmonellosis. Infect Immun. 71, 5881-5891.

630 Claudi, B., Sprote, P., Chirkova, A., Personnic, N., Zankl, J., Schurmann, N., Schmidt, A., Bumann, D., 2014. Phenotypic variation of Salmonella in host tissues delays eradication by antimicrobial chemotherapy. Cell. 158, 722-733.

Coward, C., Restif, O., Dybowski, R., Grant, A.J., Maskell, D.J., Mastroeni, P., 2014. The effects of vaccination and immunity on bacterial infection dynamics in vivo. PLoS Pathog. 10, e1004359.

Crimmins, G.T., Isberg, R.R., 2012. Analyzing microbial disease at high resolution: following the fate of the bacterium during infection. Curr Opin Microbiol. 15, 23-27.

Crump, J.A., 2012. Typhoid Fever and the challenge of nonmalaria febrile illness in sub-saharan Africa. Clin Infect Dis. 54, 1107-1109.

Crump, J.A., Luby, S.P., Mintz, E.D., 2004. The global burden of typhoid fever. Bull World Health 640 Organ. 82, 346-353.

Crump, J.A., Mintz, E.D., 2010. Global trends in typhoid and paratyphoid Fever. Clin Infect Dis. 50, 241-246.

Crump, J.A., Sjolund-Karlsson, M., Gordon, M.A., Parry, C.M., 2015. Epidemiology, Clinical Presentation, Laboratory Diagnosis, Antimicrobial Resistance, and Antimicrobial Management of Invasive Salmonella Infections. Clinical microbiology reviews. 28, 901-937.

Czaplewski, L., Bax, R., Clokie, M., Dawson, M., Fairhead, H., Fischetti, V.A., Foster, S., Gilmore, B.F., Hancock, R.E., Harper, D., Henderson, I.R., Hilpert, K., Jones, B.V., Kadioglu, A., Knowles, D., Olafsdottir, S., Payne, D., Projan, S., Shaunak, S., Silverman, J., Thomas, C.M., Trust, T.J., Warn, 
P., Rex, J.H., 2016. Alternatives to antibiotics-a pipeline portfolio review. The Lancet. Infectious diseases. 16, 239-251.

Duneau, D., Ferdy, J.B., Revah, J., Kondolf, H., Ortiz, G.A., Lazzaro, B.P., Buchon, N., 2017. Stochastic variation in the initial phase of bacterial infection predicts the probability of survival in D. melanogaster. Elife. 6.

Dybowski, R., Restif, O., Goupy, A., Maskell, D.J., Mastroeni, P., Grant, A.J., 2015. Single passage in mouse organs enhances the survival and spread of Salmonella enterica. J R Soc Interface. 12, 20150702.

Everest, P., Roberts, M., Dougan, G., 1998. Susceptibility to Salmonella Typhimurium infection and effectiveness of vaccination in mice deficient in the tumor necrosis factor alpha p55 receptor. Infect Immun. 66, 3355-3364.

660 Fisher, R.A., Gollan, B., Helaine, S., 2017. Persistent bacterial infections and persister cells. Nature reviews. Microbiology. 15, 453-464.

Fraietta, I., Gasparri, F., 2016. The development of high-content screening (HCS) technology and its importance to drug discovery. Expert Opin Drug Discov. 11, 501-514.

Gjini, E., Brito, P.H., 2016. Integrating Antimicrobial Therapy with Host Immunity to Fight Drug-

Resistant Infections: Classical vs. Adaptive Treatment. PLoS Comput Biol. 12, e1004857.

Gog, J.R., Murcia, A., Osterman, N., Restif, O., McKinley, T.J., Sheppard, M., Achouri, S., Wei, B., Mastroeni, P., Wood, J.L., Maskell, D.J., Cicuta, P., Bryant, C.E., 2012. Dynamics of Salmonella infection of macrophages at the single cell level. J R Soc Interface. 9, 2696-2707.

Goh, Y.S., Grant, A.J., Restif, O., McKinley, T.J., Armour, K.L., Clark, M.R., Mastroeni, P., 2011.

670 Human IgG isotypes and activating Fcgamma receptors in the interaction of Salmonella enterica serovar Typhimurium with phagocytic cells. Immunology. 133, 74-83.

Goh, Y.S., Necchi, F., O'Shaughnessy, C.M., Micoli, F., Gavini, M., Young, S.P., Msefula, C.L., Gondwe, E.N., Mandala, W.L., Gordon, M.A., Saul, A.J., MacLennan, C.A., 2016. Bactericidal Immunity to Salmonella in Africans and Mechanisms Causing Its Failure in HIV Infection. PLoS

675 Negl Trop Dis. 10, e0004604.

Gondwe, E.N., Molyneux, M.E., Goodall, M., Graham, S.M., Mastroeni, P., Drayson, M.T., MacLennan, C.A., 2010. Importance of antibody and complement for oxidative burst and killing of invasive nontyphoidal Salmonella by blood cells in Africans. Proc Natl Acad Sci U S A. 107, 30703075.

680 Gordon, M.A., 2011. Invasive nontyphoidal Salmonella disease: epidemiology, pathogenesis and diagnosis. Current opinion in infectious diseases. 24, 484-489.

Gordon, M.A., Kankwatira, A.M., Mwafulirwa, G., Walsh, A.L., Hopkins, M.J., Parry, C.M., Faragher, E.B., Zijlstra, E.E., Heyderman, R.S., Molyneux, M.E., 2010. Invasive non-typhoid salmonellae establish systemic intracellular infection in HIV-infected adults: an emerging disease pathogenesis. Clin Infect Dis. 50, 953-962.

Grant, A.J., Foster, G.L., McKinley, T.J., Brown, S.P., Clare, S., Maskell, D.J., Mastroeni, P., 2009. Bacterial growth rate and host factors as determinants of intracellular bacterial distributions in systemic Salmonella enterica infections. Infection and immunity. 77, 5608-5611.

Grant, A.J., Morgan, F.J., McKinley, T.J., Foster, G.L., Maskell, D.J., Mastroeni, P., 2012. Attenuated

690 Salmonella Typhimurium lacking the pathogenicity island-2 type 3 secretion system grow to high bacterial numbers inside phagocytes in mice. PLoS Pathog. 8, e1003070.

Grant, A.J., Restif, O., McKinley, T.J., Sheppard, M., Maskell, D.J., Mastroeni, P., 2008. Modelling within-host spatiotemporal dynamics of invasive bacterial disease. PLoS Biol. 6, e74.

Griffin, A.J., Li, L.X., Voedisch, S., Pabst, O., McSorley, S.J., 2011. Dissemination of persistent intestinal bacteria via the mesenteric lymph nodes causes typhoid relapse. Infect Immun. 79, 14791488.

Hancock, R.E., Nijnik, A., Philpott, D.J., 2012. Modulating immunity as a therapy for bacterial infections. Nature reviews. Microbiology. 10, 243-254. 
Helaine, S., Cheverton, A.M., Watson, K.G., Faure, L.M., Matthews, S.A., Holden, D.W., 2014. Internalization of Salmonella by macrophages induces formation of nonreplicating persisters. Science. 343, 204-208.

Helaine, S., Holden, D.W., 2013. Heterogeneity of intracellular replication of bacterial pathogens. Current opinion in microbiology. 16, 184-191.

Helaine, S., Thompson, J.A., Watson, K.G., Liu, M., Boyle, C., Holden, D.W., 2010. Dynamics of intracellular bacterial replication at the single cell level. Proc Natl Acad Sci U S A. 107, 3746-3751. Kaiser, P., Regoes, R.R., Dolowschiak, T., Wotzka, S.Y., Lengefeld, J., Slack, E., Grant, A.J., Ackermann, M., Hardt, W.D., 2014. Cecum lymph node dendritic cells harbor slow-growing bacteria phenotypically tolerant to antibiotic treatment. PLoS Biol. 12, e1001793.

Kaiser, P., Slack, E., Grant, A.J., Hardt, W.D., Regoes, R.R., 2013. Lymph node colonization dynamics after oral salmonella typhimurium infection in mice. PLoS pathogens. 9, e1003532.

Kanvatirth, P., Rossi, O., Restif, O., Blacklaws, B.A., Tonks, P., Grant, A.J., Mastroeni, P., 2020. Dual role of splenic mononuclear and polymorphonuclear cells in the outcome of ciprofloxacin treatment of Salmonella enterica infections. J Antimicrob Chemother.

Kariuki, S., Gordon, M.A., Feasey, N., Parry, C.M., 2015. Antimicrobial resistance and management of invasive Salmonella disease. Vaccine. 33 Suppl 3, C21-C29.

Kaufmann, S.H.E., Dorhoi, A., Hotchkiss, R.S., Bartenschlager, R., 2017. Host-directed therapies for bacterial and viral infections. Nat Rev Drug Discov.

Klemm, E.J., Gkrania-Klotsas, E., Hadfield, J., Forbester, J.L., Harris, S.R., Hale, C., Heath, J.N., Wileman, T., Clare, S., Kane, L., Goulding, D., Otto, T.D., Kay, S., Doffinger, R., Cooke, F.J., 720 Carmichael, A., Lever, A.M., Parkhill, J., MacLennan, C.A., Kumararatne, D., Dougan, G., Kingsley, R.A., 2016. Emergence of host-adapted Salmonella Enteritidis through rapid evolution in an immunocompromised host. Nature microbiology. 1, 15023.

Kolter, R., Siegele, D.A., Tormo, A., 1993. The stationary phase of the bacterial life cycle. Annu Rev Microbiol. 47, 855-874.

725 Kussell, E., Leibler, S., 2005. Phenotypic diversity, population growth, and information in fluctuating environments. Science. 309, 2075-2078.

Lewis, K., 2013. Platforms for antibiotic discovery. Nature reviews. Drug discovery. 12, 371-387.

Liang-Takasaki, C.J., Saxen, H., Makela, P.H., Leive, L., 1983. Complement activation by polysaccharide of lipopolysaccharide: an important virulence determinant of salmonellae. Infection and immunity. 41, 563-569.

Lim, Y., Shiver, A.L., Khariton, M., Lane, K.M., Ng, K.M., Bray, S.R., Qin, J., Huang, K.C., Wang, B., 2019. Mechanically resolved imaging of bacteria using expansion microscopy. PLoS Biol. 17, e3000268.

MacLennan, C.A., Gondwe, E.N., Msefula, C.L., Kingsley, R.A., Thomson, N.R., White, S.A.,

Goodall, M., Pickard, D.J., Graham, S.M., Dougan, G., Hart, C.A., Molyneux, M.E., Drayson, M.T., 2008. The neglected role of antibody in protection against bacteremia caused by nontyphoidal strains of Salmonella in African children. J Clin Invest. 118, 1553-1562.

MacLennan, C.A., Saul, A., 2014. Vaccines against poverty. Proc Natl Acad Sci U S A. 111, 1230712312.

740 Maskell, D.J., Hormaeche, C.E., 1985. Relapse following cessation of antibiotic therapy for mouse typhoid in resistant and susceptible mice infected with salmonellae of differing virulence. J Infect Dis. 152, 1044-1049.

Maskell, D.J., Hormaeche, C.E., 1986. Genes within the major histocompatibility complex influence the response to ampicillin therapy and severity of relapse in H-2 congenic, susceptible Itys mice infected with virulent Salmonella Typhimurium. J Immunogenet. 13, 451-457.

Mastroeni, P., Arena, A., Costa, G.B., Liberto, M.C., Bonina, L., Hormaeche, C.E., 1991. Serum TNF alpha in mouse typhoid and enhancement of a Salmonella infection by anti-TNF alpha antibodies. Microb Pathog. 11, 33-38. 
Mastroeni, P., Clare, S., Khan, S., Harrison, J.A., Hormaeche, C.E., Okamura, H., Kurimoto, M., 750 Dougan, G., 1999. Interleukin 18 contributes to host resistance and gamma interferon production in mice infected with virulent Salmonella Typhimurium. Infect Immun. 67, 478-483.

Mastroeni, P., Grant, A., 2013. Dynamics of spread of Salmonella enterica in the systemic compartment. Microbes and infection / Institut Pasteur. 15, 849-857.

Mastroeni, P., Harrison, J.A., Chabalgoity, J.A., Hormaeche, C.E., 1996. Effect of interleukin 12 neutralization on host resistance and gamma interferon production in mouse typhoid. Infect Immun. 64, 189-196.

Mastroeni, P., Harrison, J.A., Robinson, J.H., Clare, S., Khan, S., Maskell, D.J., Dougan, G., Hormaeche, C.E., 1998. Interleukin-12 is required for control of the growth of attenuated aromaticcompound-dependent salmonellae in BALB/c mice: role of gamma interferon and macrophage activation. Infect Immun. 66, 4767-4776.

Mastroeni, P., Rossi, O., 2016. Immunology, epidemiology and mathematical modelling towards a better understanding of invasive non-typhoidal Salmonella disease and rational vaccination approaches. Expert review of vaccines. 15, 1545-1555.

Mastroeni, P., Skepper, J.N., Hormaeche, C.E., 1995. Effect of anti-tumor necrosis factor alpha antibodies on histopathology of primary Salmonella infections. Infect Immun. 63, 3674-3682.

Mastroeni, P., Vazquez-Torres, A., Fang, F.C., Xu, Y., Khan, S., Hormaeche, C.E., Dougan, G., 2000. Antimicrobial actions of the NADPH phagocyte oxidase and inducible nitric oxide synthase in experimental salmonellosis. II. Effects on microbial proliferation and host survival in vivo. J Exp Med. 192, 237-248.

770 Mastroeni, P., Villarreal-Ramos, B., Hormaeche, C.E., 1992. Role of T cells, TNF alpha and IFN gamma in recall of immunity to oral challenge with virulent salmonellae in mice vaccinated with live attenuated aro- Salmonella vaccines. Microb Pathog. 13, 477-491.

Mastroeni, P., Villarreal-Ramos, B., Hormaeche, C.E., 1993. Effect of late administration of antiTNF alpha antibodies on a Salmonella infection in the mouse model. Microb Pathog. 14, 473-480. Mc, D.W., 1958. Microbial persistence. The Yale journal of biology and medicine. 30, 257-291.

Meynell, G.G., Stocker, B.A., 1957. Some hypotheses on the aetiology of fatal infections in partially resistant hosts and their application to mice challenged with Salmonella Paratyphi-B or Salmonella Typhimurium by intraperitoneal injection. J Gen Microbiol. 16, 38-58.

Micoli, F., Rondini, S., Alfini, R., Lanzilao, L., Necchi, F., Negrea, A., Rossi, O., Brandt, C., Clare, 780 S., Mastroeni, P., Rappuoli, R., Saul, A., MacLennan, C.A., 2018. Comparative immunogenicity and efficacy of equivalent outer membrane vesicle and glycoconjugate vaccines against nontyphoidal Salmonella. Proc Natl Acad Sci U S A. 115, 10428-10433.

Mosser, D.M., Edwards, J.P., 2008. Exploring the full spectrum of macrophage activation. Nature reviews. Immunology. 8, 958-969.

785 Moxon, E.R., Murphy, P.A., 1978. Haemophilus influenzae bacteremia and meningitis resulting from survival of a single organism. Proc Natl Acad Sci U S A. 75, 1534-1536.

Muotiala, A., Makela, P.H., 1990. The role of IFN-gamma in murine Salmonella Typhimurium infection. Microb Pathog. 8, 135-141.

Okoro, C.K., Kingsley, R.A., Quail, M.A., Kankwatira, A.M., Feasey, N.A., Parkhill, J., Dougan, G., 790 Gordon, M.A., 2012. High-resolution single nucleotide polymorphism analysis distinguishes recrudescence and reinfection in recurrent invasive nontyphoidal Salmonella Typhimurium disease. Clin Infect Dis. 54, 955-963.

Oshota, O., Conway, M., Fookes, M., Schreiber, F., Chaudhuri, R.R., Yu, L., Morgan, F.J.E., Clare, S., Choudhary, J., Thomson, N.R., Lio, P., Maskell, D.J., Mastroeni, P., Grant, A.J., 2017. 795 Transcriptome and proteome analysis of Salmonella enterica serovar Typhimurium systemic infection of wild type and immune-deficient mice. PLoS One. 12, e 0181365.

Pham, T.H.M., Brewer, S.M., Thurston, T., Massis, L.M., Honeycutt, J., Lugo, K., Jacobson, A.R., Vilches-Moure, J.G., Hamblin, M., Helaine, S., Monack, D.M., 2020. Salmonella-Driven 
Polarization of Granuloma Macrophages Antagonizes TNF-Mediated Pathogen Restriction during Persistent Infection. Cell Host Microbe. 27, 54-67 e55.

Price, D.J., Breuze, A., Dybowski, R., Mastroeni, P., Restif, O., 2017. An efficient moments-based inference method for within-host bacterial infection dynamics. PLoS Comput Biol. 13, e1005841. Ramachandran, G., Boyd, M.A., MacSwords, J., Higginson, E.E., Simon, R., Galen, J.E., Pasetti, M.F., Levine, M.M., Tennant, S.M., 2016. Opsonophagocytic Assay To Evaluate Immunogenicity of Nontyphoidal Salmonella Vaccines. Clin Vaccine Immunol. 23, 520-523.

Richter-Dahlfors, A., Buchan, A.M., Finlay, B.B., 1997. Murine salmonellosis studied by confocal microscopy: Salmonella Typhimurium resides intracellularly inside macrophages and exerts a cytotoxic effect on phagocytes in vivo. J Exp Med. 186, 569-580.

Richter-Dahlfors, A., Buchan, A.M.J., Finlay, B.B., 1997. Murine salmonellosis studied by confocal microscopy: Salmonella Typhimurium resides intracellularly inside macrophages and exerts a cytotoxic effect on phagocytes in vivo. J Exp Med. 186, 569-580.

Rollenhagen, C., Sorensen, M., Rizos, K., Hurvitz, R., Bumann, D., 2004. Antigen selection based on expression levels during infection facilitates vaccine development for an intracellular pathogen. Proc Natl Acad Sci U S A. 101, 8739-8744.

815 Rossi, O., Coward, C., Goh, Y.S., Claassens, J.W.C., MacLennan, C.A., Verbeek, S.J., Mastroeni, P., 2019. The essential role of complement in antibody-mediated resistance to Salmonella. Immunology. 156, 69-73.

Rossi, O., Dybowski, R., Maskell, D.J., Grant, A.J., Restif, O., Mastroeni, P., 2017. Within-host spatiotemporal dynamics of systemic Salmonella infection during and after antimicrobial treatment.

820 J Antimicrob Chemother. 72, 3390-3397.

Rossi, O., Grant, A.J., Mastroeni, P., 2017. Effect of in vivo neutralization of tumor necrosis alpha on the efficacy of antibiotic treatment in systemic Salmonella enterica infections. Pathog Dis. 75.

Salcedo, S.P., Noursadeghi, M., Cohen, J., Holden, D.W., 2001. Intracellular replication of Salmonella Typhimurium strains in specific subsets of splenic macrophages in vivo. Cell Microbiol.

8253 3, 587-597.

Saliba, A.E., Li, L., Westermann, A.J., Appenzeller, S., Stapels, D.A., Schulte, L.N., Helaine, S., Vogel, J., 2016. Single-cell RNA-seq ties macrophage polarization to growth rate of intracellular Salmonella. Nat Microbiol. 2, 16206.

Schwartz, D.J., Chen, S.L., Hultgren, S.J., Seed, P.C., 2011. Population dynamics and niche 830 distribution of uropathogenic Escherichia coli during acute and chronic urinary tract infection. Infect Immun. 79, 4250-4259.

Shehata, T.E., Marr, A.G., 1971. Effect of nutrient concentration on the growth of Escherichia coli. J Bacteriol. 107, 210-216.

Sheppard, M., Webb, C., Heath, F., Mallows, V., Emilianus, R., Maskell, D., Mastroeni, P., 2003.

835 Dynamics of bacterial growth and distribution within the liver during Salmonella infection. Cellular microbiology. 5, 593-600.

Stapels, D.A.C., Hill, P.W.S., Westermann, A.J., Fisher, R.A., Thurston, T.L., Saliba, A.E., Blommestein, I., Vogel, J., Helaine, S., 2018. Salmonella persisters undermine host immune defenses during antibiotic treatment. Science. 362, 1156-1160.

840 van Elsland, D.M., Pujals, S., Bakkum, T., Bos, E., Oikonomeas-Koppasis, N., Berlin, I., Neefjes, J., Meijer, A.H., Koster, A.J., Albertazzi, L., van Kasteren, S.I., 2018. Ultrastructural Imaging of Salmonella-Host Interactions Using Super-resolution Correlative Light-Electron Microscopy of Bioorthogonal Pathogens. Chembiochem.

Vazquez-Torres, A., Jones-Carson, J., Baumler, A.J., Falkow, S., Valdivia, R., Brown, W., Le, M., 845 Berggren, R., Parks, W.T., Fang, F.C., 1999. Extraintestinal dissemination of Salmonella by CD18expressing phagocytes. Nature. 401, 804-808.

Vazquez-Torres, A., Jones-Carson, J., Mastroeni, P., Ischiropoulos, H., Fang, F.C., 2000. Antimicrobial actions of the NADPH phagocyte oxidase and inducible nitric oxide synthase in 
experimental salmonellosis. I. Effects on microbial killing by activated peritoneal macrophages in vitro. J Exp Med. 192, 227-236.

Vazquez-Torres, A., Xu, Y., Jones-Carson, J., Holden, D.W., Lucia, S.M., Dinauer, M.C., Mastroeni, P., Fang, F.C., 2000. Salmonella pathogenicity island 2-dependent evasion of the phagocyte NADPH oxidase. Science. 287, 1655-1658.

Vlazaki, M., Huber, J., Restif, O., 2019. Integrating mathematical models with experimental data to investigate the within-host dynamics of bacterial infections. Pathog Dis. 77.

Westermann, A.J., Forstner, K.U., Amman, F., Barquist, L., Chao, Y., Schulte, L.N., Muller, L., Reinhardt, R., Stadler, P.F., Vogel, J., 2016. Dual RNA-seq unveils noncoding RNA functions in host-pathogen interactions. Nature. 529, 496-501.

Westermann, A.J., Gorski, S.A., Vogel, J., 2012. Dual RNA-seq of pathogen and host. Nature reviews. Microbiology. 10, 618-630.

Withanage, G.S., Wigley, P., Kaiser, P., Mastroeni, P., Brooks, H., Powers, C., Beal, R., Barrow, P., Maskell, D., McConnell, I., 2005. Cytokine and chemokine responses associated with clearance of a primary Salmonella enterica serovar Typhimurium infection in the chicken and in protective immunity to rechallenge. Infect Immun. 73, 5173-5182. 